\title{
A materials demand model with ordering quantity of past and recency of ordering time
}

\author{
Hui Hsin Huang \\ 32,Zhenli St., Tamsui Dist., New Taipei City, Taiwan
}

\begin{abstract}
This paper proposes a statistic model to forecast the materials demand quantity of downstream manufactures in manufacturing process. The author considers the ordering quantity of past record pattern following the normal distribution and the recency of ordering time is a renew process of statistic. The empirical data are used to estimate the parameters of proposed model and its validation. The results can be applied to predict different kind of materials demand
\end{abstract}

\section{Introduction}

In the manufacturing process, it is an important issue for downstream manufactures to predict the demand quantities of materials form upstream firm [1-3]. When forecasting material ordering quantity, downstream manufactures can avoid overproduction or product shortages and can also save the cost of inventory $[2,4]$. Thus this paper proposes a stochastic model for detecting the materials demand quantity before the upstream firm ordering

For the purpose of this model, the information of past ordering is one of the crucial factors that may impact the probability of future ordering quantity $[1,4]$. The materials consuming is often stable or following some regular pattern. Therefore, it can provide a baseline reference when predicting demand quantity in the next time. Thus, we include this variable in our model [3].

The concept of recency of ordering time stands on the view of point in RFM which is a method used for analyzing customer value [5-9]. $\mathrm{R}$ index is to explore how recently the customer makes his purchase behavior $[5,8,9]$. $\mathrm{F}$ index is to discuss how often this customer purchase [6-7]. $\mathrm{R}$ index is demonstrated by how much money this customer spends $[6,8,9]$. We extend the $\mathrm{R}$ index to the recency of ordering time which is the time length between the last ordering (purchase) time from the upstream in the observation period and the observing deadline [8]. The recency of ordering time reveals the clue that upstream manufactures may gradually run out or use up their inventory of materials if the recency of ordering time is longer[2,4,8]. This information can help the downstream manufactures to predict the demand quantity they need. Then, we consider this variable into our model.

The materials demand model is derived by the ordering quantity of past and the recency of ordering time. The paper is organized as follows. In the next section, we develop the materials demand model. We will derive the cumulative distribution function (cdf) and the probability density function (pdf) of this model with combining the ordering quantity of past and and the recency of ordering time. In Section 3, the empirical data analysis is demonstrated. It shows the results of parameter estimation and validation of real data. Finally, we offer our conclusions in section 4 .

\section{The Materials Demand Model}

We consider the materials demand model is composed by the ordering quantity of past (denoted as Q) and the recency of ordering time (denoted as R).

$$
D=R \cdot Q
$$

\subsubsection{The Ordering Quantity of Past.}

We consider the past ordering quantities(denoted as Q) is a random variable(denoted as q) which follows lognormal distribution with the parameters of $\mu, \sigma^{2}$ and its probability density function(pdf) is

$$
f_{Q}\left(q \mid \mu, \sigma^{2}\right)=\frac{1}{q \sigma \sqrt{2 \pi}} \exp \left[-\frac{(\log q-\mu)^{2}}{2 \sigma^{2}}\right]
$$

Then $\log Q$ follows normal distribution and its pdf is

$$
f_{\log Q}\left(y \mid \mu, \sigma^{2}\right)=\frac{1}{\sqrt{2 \pi \sigma^{2}}} \exp \left[-\frac{(y-\mu)^{2}}{2 \sigma^{2}}\right]
$$


We consider the recency of ordering time (denoted as R) is demonstrated as renew process [9]. Then, according to the renew process in statistic [9-12], we can calculate its pdf and cumulative distribution function (cdf). First, we assume the purchase interval time is a random variable which follows exponential distribution [8]. After that, the result of pdf and cdf in the recency of ordering time (denoted as r) are computing as

$$
f_{R}(r \mid \theta)=\frac{1}{\theta^{2}} \exp \left(-\frac{r}{\theta}\right)
$$

and

$$
F_{R}(r \mid \theta)=\frac{1-\exp \left(-\frac{r}{\theta}\right)}{\theta}
$$

\subsubsection{The Cumulative Distribution Function of Materials Demand Model.}

According to equation (1), the materials demand model is composed by "the ordering quantity of past(Q)" multiplying "the recency of ordering time(R)". In order to calculate the cdf of full model, we denote random variable $\mathrm{X}$ as $\log \mathrm{D}$ and is demonstrated in (6).

$$
X \equiv \log D=\log R+\log Q
$$

Then, its cdf is

$$
\begin{aligned}
F_{X}(x) & =P(\log R+\log Q<x) \\
& =\int_{0}^{\infty} P(\log R+\log Q \mid \log R=z, \log Q=y) f_{\log R}(z \mid \theta) d z \\
& =\int_{0}^{\infty} P(\log Q<x-\log R) f_{\log R}(z \mid \theta) d z \\
& =\int_{0}^{\infty} \int_{-\infty}^{x-r} \frac{1}{\sqrt{2 \pi \sigma^{2}}} \exp \left[-\frac{(y-\mu)^{2}}{2 \sigma^{2}}\right] d y f_{\log R}(z \mid \theta) d z \\
& =\int_{0}^{\infty} \int_{-\infty}^{\infty} \Phi\left(\frac{x-r-\mu}{\sigma}\right) d y f_{\log R}(z \mid \theta) d z
\end{aligned}
$$

In equation (7), the pdf of $\log \mathrm{R}$ can be derived by equation (4).Then its pdf, $f_{\log R}(z \mid \theta)$, is

$$
\begin{aligned}
f_{\log R}(z \mid \theta) & =\frac{d}{d z} F_{\log R}(z \mid \theta) \\
= & \frac{d}{d z} P(\log R<z) \\
= & \frac{d}{d z} P\left(\log \theta^{-2}-\frac{r}{\theta}<z\right) \\
= & \frac{d}{d z} P\left(r<\theta \log \theta^{-2}-\theta z\right)
\end{aligned}
$$

$$
\begin{aligned}
& =\frac{d}{d z}\left[\frac{1}{\theta} \int_{0}^{\theta \log \theta^{-2}-\theta z} \frac{1}{\theta} \exp -\frac{r}{\theta} d r\right] \\
= & \frac{d}{d z}\left\{\frac{1}{\theta}\left[1-\exp \left(\theta \log \theta^{-2}-\theta z\right)\right]\right\} \\
= & \exp \left(\theta \log \theta^{-2}-\theta z\right)
\end{aligned}
$$

\subsubsection{The Probability Density Function of} Materials Demand Model. Based on equation (7), we can compute the pdf of the proposed model. It shows in equation (9).

$$
f_{X}(x)=\frac{d}{d x} F_{X}(x)=\frac{d}{d x} \int_{0}^{\infty} \int_{-\infty}^{\infty} \Phi\left(\frac{x-r-\mu}{\sigma}\right) \exp \left(\theta \log \theta^{-2}-\theta z\right) d y d z
$$

\section{The Empirical Data Analysis}

In the empirical data analysis, we divide the empirical data into two parts. First parts are used to estimate the parameters of the proposed model. Then, according to the results of parameters estimation, the simulation data are obtained. Finally, we calculate the root-mean-square deviation (RMSD) to show the results of comparison between empirical data and simulation data.

The analysis process is demonstrated in Figure 1.

Stept1:

To divide the empirical data into two parts

$\begin{aligned} & \text { Stept2: } \\ & \text { To estimate the parameters of } \\ & \text { model based on partial data }\end{aligned}$
$\begin{aligned} & \text { Stept3: } \\ & \text { To get the simulation data based } \\ & \text { on the results of parameters } \\ & \text { estimation }\end{aligned}$
$\begin{aligned} & \text { Stept4: } \\ & \text { To compute RMSD from the } \\ & \text { comparison between partial data } \\ & \text { and simulation data }\end{aligned}$

Figure 1. The steps of analysis

3.1.1 The Parameters estimation. We use MLE (maximum likelihood estimate) to estimate the parameters. Let $\mathrm{x}_{\mathrm{j}}$ denote the materials demand quantities by upstream $\mathrm{j}$, that is: 


$$
L\left(\mu, \sigma^{2}, \theta\right)=\prod_{j=1}^{n} f_{X}\left(x_{j}\right)=\frac{d}{d x} \int_{0}^{\infty} \int_{-\infty}^{\infty}\left[\Phi\left(\frac{x-r-\mu}{\sigma}\right)\right]^{n} \exp \left[n \theta\left(\log \theta^{-2}-z\right)\right] d y d z
$$

We differentiate $L\left(\mu, \sigma^{2}, \theta\right)$ respectively regarding $\mu, \sigma^{2}, \theta$ and set them equal to zero.

That is,

$$
\left\{\begin{array}{l}
\frac{\partial \mu}{\partial} L_{j}\left(\mu, \sigma^{2}, \theta\right)=\frac{d}{d x} \int_{0}^{\infty} \int_{-\infty}^{\infty} \Phi\left(\frac{x-r-\mu}{\sigma}\right) \frac{n(y-\mu)}{\sigma^{2}} \exp \left[n \theta\left(\log \theta^{-2}-z\right)\right] d y d z=0 \\
\frac{\partial \sigma^{2}}{\partial} L_{j}\left(\mu, \sigma^{2}, \theta\right)=\frac{d}{d x} \int_{0}^{\infty} \int_{-\infty}^{\infty} \Phi\left(\frac{x-r-\mu}{\sigma}\right) \frac{4 n(y-\mu)^{2}}{\sigma^{3}} \exp \left[n \theta\left(\log \theta^{-2}-z\right)\right] d y d z=0 \\
\frac{\partial \theta}{\partial} L_{j}\left(\mu, \sigma^{2}, \theta\right)=\frac{d}{d x} \int_{0}^{\infty} \int_{-\infty}^{\infty} \Phi\left(\frac{x-r-\mu}{\sigma}\right) \exp \left[n \theta\left(\log \theta^{-2}-z\right)\right]\left[n\left(\log \theta^{-2}-2-z\right)\right] d y d z=0
\end{array}\right.
$$

The estimation results show in table1.

Table 1. The results of estimation.

\begin{tabular}{|c|c|}
\hline Parameters & \\
\hline$\mu$ & 320 \\
\hline$\sigma^{2}$ & 2.356 \\
\hline$\theta$ & 43.27 \\
\hline
\end{tabular}

3.1.2 The Model Valiadation. We calculate the root-mean-square deviation (RMSD) to make comparison between empirical data and simulation data. The result shows in table 2 .

Table 2. The results of RMSD.

$$
\begin{array}{c|c|}
\hline \text { RMSD }^{*} & \mathbf{0 . 8 7 9} \\
{ }^{*} \mathrm{RMSD} & =\frac{\sqrt{\sum_{i=1}^{m}\left(v_{i}-\hat{v}_{i}\right)^{2}}}{m}
\end{array}
$$

$m$ is the simple size

$v_{i}$ is the $i$-th empirical data

$\hat{v}_{i}$ is the $i$-th simulation data

The results of RMSD is smaller than 1. Then the proposed model has acceptable fitness with real data.

\section{The Conclusion}

This paper proposes a stochastic model to forecast the materials demand quantities. Different from previous researches, author considers the recency of ordering time as a factor to detect how long the upstream firm does not make ordering. This may impact its quantities demand but rarely be included in the demand model developing process.

In the future, researches can explore other probability distributions to try fitness between model and real data to improve the predictive power. For example, researchers can consider Weibull distribution or Erlang distributioin to instead of exponential of the recency variable.

\section{Acknowledgements}

The author would like to thank the National Science Council of Taiwan (105WFDA450005) and Aletheia University (AU-AR-104-014) for supporting this research.

\section{References}

1. Y. Xia, Responding to supplier temporary price discounts in a supply chain through ordering and pricing decisions, Int. J. Prod. Res. (2015)1-13.

2. H. H. Huang, A Predicting Model for Demand Quantities of Downstream Manufacturer, Applied Mechanics and Materials 624(2014) 694-697.

3. H. Kim, J. C. Lu, P. H. Kvam1, Y.C. Tsao, Ordering quantity decisions considering uncertainty in supply-chain logistics operations, Int. J. Prod. Econ. 134(2011) 16-27.

4. S. C. Chen, L. E. Cárdenas-Barrón, J. T. Teng, Retailer's economic order quantity when the supplier offers conditionally permissible delay in payments link to order quantity, Int. J. Prod. Econ.155 (2014) 284-291.

5. E. Nikumanesh, A. Albadvi, Customer's life-time value using the RFM model in the banking industry: a 
case study, Int. J. Elec. Cust. Relat. Manag. 8(2014)234-245.

6. J. H. Kao , F. Lai, H. T. Liaw, P.H. Hsieh, Study of Customer Value and Supplier Dependence with the RFM Model, Lecture Notes in Electrical Engineering331(2015) 283-296.

7. K. Coussement, F. A.M. Van den Bossche, K. W. De Bocka, Data accuracy's impact on segmentation performance: Benchmarking RFM analysis, logistic regression, and decision trees, J. Bus. Res. 67 (2014) 2751-2758.

8. H. H. Huang, A detection model of customer alive in information management application, Adv. Mater. Res.684 (2013) 505-508.
9. Y. S. Cho, S. C. Moon, S. Jeong, I.B. Oh, K. H. Ryu, Clustering Method Using Item Preference Based on RFM for Recommendation System in U-Commerce, Ubiquitous Information Technologies and Applications 214 (2013) 353-362.

10. M.S. Ross, Stochastic Process, Wiley, New York, (1995).

11. M.S. Ross, Applied Probability Models with Optimization Applications, Dover Publication, New York, (2013).

12. M.S. Ross, Introduction to Stochastic Dynamic Programming, Academic Press, London, (2014) 